\title{
WIR SIND HALBIERTE. DIE ENTDECKUNG DER DDR IN DER WESTDEUTSCHEN LITERATUR VOR 1989
}

\begin{abstract}
We are bisected. Discovering the GDR in West German Literature before 1989
West German literature has turned its back to the existence of the second German state until the 1980s. Only a few years before the fall of the Berlin wall, three writers started to make the GDR a subject of narration or poetry: Botho Strauß, Peter Schneider and Martin Walser. In different ways, yet unanimously, they complain about the division of Germany dealing with its impact on everyday life and private feelings.

KEY WORDS: Division of Germany, West German literature during the 1980s
\end{abstract}

Viel war es nicht. Aber wenig.

Martin Walser: Dorle und Wolf

In der (Roman-)Literatur der DDR ist die deutsche Teilung von Anfang an präsent gewesen. Um die gesellschaftliche Gegenwart, dem Prinzip des Sozialistischen Realismus folgend, in ihrer dialektischen Dynamik zu erfassen, sind die erkannten Widersprüche als 'typische' Konflikte mit 'objektiver' Tendenz zu gestalten gewesen; unter den politisch-sozialen Gegebenheiten der DDR hieß das in erster Linie, die Rivalität mit dem anderen, kapitalistischen Deutschland in klarer Frontziehung zum Ausdruck zu bringen, damit den Lesern ihre Identifikation mit dem eigenen Staat alternativlos wurde. Umso besser konnte die politisch in Verantwortung genommene Literatur vorbildhaft wirken und den marxistisch-leninistisch begründeten Fortschrittsoptimismus durch glücklich endende Handlungen bestätigen, als sich die ökonomischen Probleme in der Fiktion weit überzeugender einer Lösung im Sinne der SED zuführen ließen als in der Lebenswelt. In der DDR hat es daher ,spätestens seit Eduard Claudius' Schule machendem Aufbauroman Menschen an unserer Seite (1951) kaum einen zeitgeschichtlichen Roman“ gegeben, „der nicht in wesentlichen 
Teilen auch in der Bundesrepublik spielen würde und den Gegensatz der politischen, ökonomischen und sozialen Systeme zum Thema hätte" (Meier, 1998, S. 62).

Fraglos ist dieses entschieden parteiliche Erzählen von der deutsch-deutschen Konkurrenz nach stets dem gleichen Gut/Böse-Schema organisiert: Die spätkapitalistische Bundesrepublik, wo die faschistoide Reaktion angeblich am Ruder geblieben ist, steht für das historisch falsifizierte Alte; der DDR hingegen gehört die humane Zukunft, da sie aus dem Zusammenbruch des Nationalsozialismus die einzig richtige Konsequenz der Verstaatlichung gezogen hat (Hutchinson, 2011 [zuerst 1977]; Grundmann, 1988). 'Typischster' Vertreter westdeutscher Interessen ist daher der in zahlreichen DDR-Romanen sein Unwesen treibende 'Sabogent', der aus ideologischer Verblendung oder egoistischer Skrupellosigkeit den unaufhaltsamen Erfolg des SED-Sozialismus behindern will und zu diesem Zweck vor Verbrechen nicht zurückschreckt, bevor ihn loyale DDR-Bürger (unter tätiger Mithilfe der Partei) zu enttarnen wissen. In diese Topik gehört ebenso unvermeidlich das stereotype Motiv der Republikflucht: Entweder trennt sie in ihrer negativen Variante die kapitalistische Spreu vom sozialistischen Weizen, indem unbelehrbare Ost-Charaktere in ihre geistige Heimat verschwinden, oder sie führt in positivem Sinn zur reumütigen Besinnung auf die Sache des Sozialismus, indem die Konfrontation mit dem wirtschaftlich überlegenen, doch menschlich defizienten Westen labile Protagonisten auf den richtigen Weg zurückverweist. Noch Christa Wolfs Der geteilte Himmel (1963) funktioniert dem sozialistisch-realistischen Muster gemäß, wenn der kurze Roman die so vielversprechende Liebe zwischen der 19-jährigen Rita Seidel und dem wesentlich älteren Manfred Herrfurth parabelhaft scheitern lässt: Der Sohn aus großbürgerlicher Familie erliegt der egozentrischen Verlockung einer Karriere im Westen, während das Mädchen vom Land in der kollektiven Produktion heranreift und bei aller naiven Freude an der Warenpracht Westberlins deren moralische Leere durchschaut. Es ist daher der Mauerbau 1961, der Ritas Kontakt zu Manfred unterbindet und auf Dauer dafür sorgt, die ethisch-ideologisch-historisch zwingende Entscheidung zu treffen, wie existenziell einschneidend diese Trennung auch immer verlaufen mag.

Steht die deutsche Zweistaatlichkeit insofern auch während der 1960er Jahre ganz im Zentrum der DDR-Literatur, so kommt sie auffälliger Weise gerade bei denjenigen westdeutschen Autoren kaum zur Sprache, die sich als Kritiker der 'Adenauerschen Restauration' verstanden, der Bundesrepublik als Nonkonformisten in distanzierter Skepsis begegneten und nicht darauf vertrauen mochten, dass es in Westdeutschland zu einem wahrhaften demokratischen Gemeinwesen kommen würde (Meier, 1998, S. 59-61). Stand jenseits der Elbe der sozialistisch verordnete Optimismus im Vordergrund, so dominierte diesseits ein entschiedener Pessimismus, der seinen geradezu emblematischen Ausdruck in Wolfgang Koeppens 'Trilogie des Scheiterns' Tauben im Gras (1951), Das Treibhaus (1953) und Der Tod in Rom (1954) fand (Meier, 2003, S. 135-149) und der primär ästhetizistischen 
Ausrichtung (in Hinsicht auf Wolfgang Koeppen vgl. Meier, 2006, S. 202-216) wegen gar nicht daran interessiert zu sein brauchte, die politisch-historische Realität wirklichkeitsgetreu zu schildern. Ganz offensichtlich bestand auf nonkonformistischer Seite ein geringerer Bedarf an der Reflexion realpolitischer Gegebenheiten im Gefolge des Nationalsozialismus als an der Thematisierung der eigenen künstlerisch-antibürgerlichen Befindlichkeit. Das Fazit für die ersten beiden Nachkriegsjahrzehnte steht daher außer Frage:

Auf das Faktum der Zweistaatlichkeit mit allen Folgeerscheinungen lassen sich zunächst - wenn überhaupt - nur solche Autoren der Bundesrepublik ein, die dem konservativen bis reaktionären Spektrum angehören und ihre literarische Arbeit als Beitrag zum Kampf gegen den Kommunismus verstehen (als ihr namhaftester Vertreter kann Rudolf Krämer-Badoni gelten). (Meier, 1998, S. 61)

Dass es mit der DDR bzw. der 'Ostzone' seit 1949 einen zweiten deutschen Staat gegeben hat, ist vor 1980 - von wenigen und letztlich marginalen Erwähnungen etwa in Günter Grass' Hundejahre (1963) abgesehen - lediglich in einem humoristischen Kurzroman handlungstragend gewesen: In Arno Schmidts Das steinerne Herz (1956) begibt sich ein westdeutscher Büchernarr in die DDR, um in der Staatsbibliothek Berlin (Ost) seine Doublette der zweiten Auflage eines statistischen Werks über das Königreich Hannover 'zwangsweise' gegen die begehrte dritte Auflage auszutauschen; eher nebenbei verhilft er zugleich der Geliebten eines Bekannten zur illegalen Übersiedelung in den Westen. Wenn es vor diesem Hintergrund zu erstaunlich wohlmeinenden Urteilen des Ich-Erzählers über die Lebensverhältnisse im Osten kommt, wo die „Leute (...) so rührend ehrlich arbeiten; weil sie so tapfer gottlos sind" (Schmidt, 1956, S. 181), dann wird das wesentlich durch persönliche Ressentiments des Intellektuellen motiviert, der die Geistlosigkeit des gegenwärtigen Alltags hüben wie drüben perhorresziert und der kulturellen Vitalität der Weimarer Republik nachtrauert: „was mich neugierig in die DDR führt, ist weniger die anziehende Kraft des Ostens - den ich ja kaum noch kenne! - als vielmehr die abstoßende des Westens!“ (Schmidt, 1956, S. 135). Wie wenig sich dieses sarkastische Erzählen auf die faktische Lebenswirklichkeit jenseits der Grenze einlassen will, zeigt nicht zuletzt die Tatsache, dass der Volksaufstand vom 17. Juni 1953 im laut Untertitel ,,dem Jahre 1954 nach Christi” entstammenden 'historischen Roman' keinerlei Erwähnung findet.

Während der 1960/70er Jahre ist das Thema 'DDR' dann sogar vollständig aus dem Themen-Horizont der westdeutschen Literatur verschwunden, sofern man nicht den 1959 übersiedelten Uwe Johnson als Sonderfall in Rechnung stellen will. Die politisch-gesellschaftlichen Besonderheiten im sozialistischen Deutschland kommen für bundesrepublikanische Leser seinerzeit allein durch auch im Westen populär gewordene Romane von Ost-Autoren zur Geltung: namentlich in Hermann Kants bei aller Witzelei dezidiert linientreuem Bildungsroman Die Aula (1965) sowie in fraglos reflektierterer Brechung bei Christa Wolf seit Nachdenken über Christa T. 
(1968). Infolgedessen gilt es für die westdeutschen Autoren geradezu von einer literarischen 'Hallstein-Doktrin' (Kilian, 2001) zu sprechen, die den völkerrechtlichen Alleinvertretungsanspruch der Bundesrepublik - in einer Regierungserklärung vom 29. 9. 1955 erhoben - poetisch durchsetzt. Die entsprechende Wirklichkeitsabstinenz künstlerisch ambitionierter Schriftsteller sollte sich bis in die 1980er Jahre und damit bis in die unmittelbare Vorgeschichte des Mauerfalls am 9. November 1989 - hinein behaupten.

Zuvor hat die seit 1969 betriebene 'Ostpolitik' der sozialliberalen Bundesregierung unter Willy Brandt allein in der deutschsprachigen Pop-Musik ein punktuelles Echo gefunden: Udo Lindenbergs Wir wollen doch nur zusammen sein (Mädchen aus Ost-Berlin), 1973 auf der Debüt-LP Alles klar auf der Andrea Doria veröffentlicht, lässt einen jungen Mann aus dem Westen in Ost-Berlin auf „ein ganz heißes Mädchen aus Pankow“ treffen und gemeinsam mit ihr ,,von einem Rock-Festival / auf dem Alexanderplatz / mit den Rolling Stones und 'ner Band aus Moskau“ träumen, bevor sie ihn darauf aufmerksam macht, dass er ja ,spätestens um zwölf / wieder drüben sein“ muss: „sonst gibt's die größten Nervereien / denn du hast ja nur'n Tagesschein“. Dieses An- (bzw. Aus-)sprechen der Regularien im damaligen West/Ost-Kontakt ist als ein 'effet de réel' im Sinne von Roland Barthes (2002) zu verstehen, dessen Konkretheit der immer schon auf Realien ausgerichteten Pop-Kultur leichter fällt als der artistisch-autonomen Hochkultur und daher auch zwanglos in den Appell an politische Veränderung übergehen kann:

\footnotetext{
ich mußte gehen, obwohl ich so gerne noch geblieben wär / aber Mädchen, ich komme wieder / und vielleicht geht's auch irgendwann mal ohne Nervereien / da muß doch auf die Dauer was zu machen sein! / Ich hoffe, daß die Jungs das nun bald in Ordnung bringen / denn wir wollen doch einfach nur zusammen sein / vielleicht auch mal etwas länger / vielleicht auch mal etwas enger / wir wollen doch einfach nur zusammen sein (Lindenberg, 2002, o. S.)
}

Udo Lindenbergs Lied über die privaten Folgen der politischen Teilung ist kein Hit gewesen. Erst recht hat die darin formulierte Hoffnung, auch zwischen den beiden deutschen Staaten könnte es bald einmal 'etwas enger' zugehen, im Bewusstsein der westdeutschen Intellektuellen-Kultur auf lange Jahre keinerlei Widerhall gefunden. Vielmehr bleibt zu konstatieren, dass das Interesse an einer - wie immer gearteten - Annäherung beider gesellschaftlichen Systeme gerade im Gefolge der offensichtlichen 'Entspannung' bis in die 1980er Jahre hinein erloschen war. Geändert hat sich dieses Faktum erst, als sich gerade von 'linker', d. h. nicht nationalistisch motivierter Seite ein spätes Bedürfnis meldete, die Teilung nun doch zum problematischen Motiv zu machen und mit den Mitteln der Poesie darauf einzugehen. Udo Lindenbergs Erfolgssingle Sonderzug nach Pankow (am 2. Februar 1983 veröffentlicht) auf die Melodie von Glenn Millers Chattanooga Choo Choo ist dafür symptomatisch: In westlich-lässiger Kumpelhaftigkeit apostrophiert der Sänger den Staatsratsvorsitzenden Erich Honecker und verlangt danach, wie so viele 'Schlager- 
affen' aus der Bundesrepublik einmal in Ostberlin auftreten zu können (dazu ist es am 25. Oktober 1983 in der Tat gekommen). Der 1946 im westfälischen Gronau geborene Udo Lindenberg kann seitdem als der Pop-Spezialist für deutsch-deutsche Befindlichkeit gelten und darf unbestritten das Verdienst für sich in Anspruch nehmen, über lange Jahre als weithin einsame Stimme die Abwegigkeit der Spaltung Deutschlands in konträre Systeme angesprochen zu haben.

Wenn sich nach gut 30 Jahren gewaltsamer Teilung die literarische Situation verändert hat, dann heißt das dennoch nicht, dass das andere Deutschland nun zu einem vielfach bearbeiteten Motiv westdeutscher Autoren geworden wäre. Deren Reaktionen auf die politische Tatsache 'DDR' bleiben weiterhin an einer Hand abzuzählen, sind jetzt aber nicht mehr wie in den Anfangsjahren das Privileg konservativer Denker fortgeschrittenen Alters. In seinem motivisch wie formal hochkomplexen Langgedicht Diese Erinnerung an einen, der nur einen Tag zu Gast war (im Sommer und Herbst 1984 verfasst und 1985 veröffentlicht) macht der 1944 in Nietzsches Ursprungsort Naumburg/Saale geborene Botho Strauß seine politikgeschichtlich prekär gewordene Identität bedenklich: „Bin ich denn nicht geboren in meinem Vaterland?" (Strauß, 1985, S. 49). In kulturkritischer Topik wird die gewissermaßen natürliche Herkunft aus einer kulturell vitalen Tradition der äußerlichen Prägung durch gesellschaftlichen Zwang entgegengesetzt:

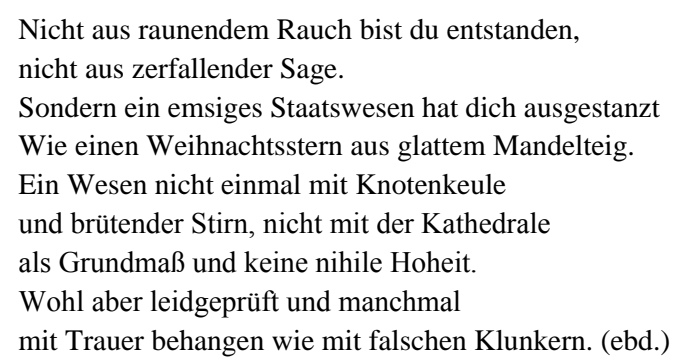

Die von einer Gedichtzeile Gottfried Benns (,Jena vor uns im lieblichen Tale“) eingeleitete Erinnerung an kindliche Spaziergänge „mit dem Vater am Ufer von Saale und Unstrut" bleibt anschlusslos („Wann war das und wo?"). Die Gegenwart ist demgegenüber von einem Bewusstsein fehlender Zugehörigkeit bestimmt, die im Gedenken an die einstige Ganzheit deutscher Kultur nur umso schmerzlicher vermisst wird:

Kein Deutschland gekannt zeit meines Lebens.

Zwei fremde Staaten nur, die mir verboten,

je im Namen eines Volkes der Deutsche zu sein.

Soviel Geschichte, um so zu enden?

Man spüre einmal: das Herz eines Kleist und

die Teilung des Lands. Man denke doch: welch ein Reunieren,

wenn einer, in uns, die Bühne der Geschichte aufschlüg! (Strauß, 1985, S. 49 f.) 
Dieser Gedankengang, der sich allein schon seiner Hochstil-Dunkelheit (Hoffmann, 2006) wegen ins Abseits der Gemeinplätze emanzipatorischer Systemkritik der 68er-Generation stellt, leitet mit dem rückhaltlosen Plädoyer für 'nationelle' Verankerung gerade des 'Dichters' (vgl. den ablehnenden Tenor der FAZ-Rezension von: Fuld, 1985, S. 24) die 1993 im Essay Anschwellender Bocksgesang kulminierende Wende von Botho Strauß zum 'Reaktionär' ein, der sich eben nicht als 'Aufhalter' oder 'Rückschrittler' begreift, sondern ,etwas Vergessenes wieder in die Erinnerung" bringen will und zuallererst „gegen Vergeßlichkeit in jeder Epoche“ kämpft (Strauß, 1990, S. 315 f.).

Mit einem biologischen Vergleich entwirft der damalige Erfolgsdramatiker eine Zukunftsperspektive, die sich aus der Rückbesinnung auf eine wesentlich kulturell motivierte Einheit ergibt und hoffen lässt, dass es gerade in der Poesie gelingen mag, doch wiederum Gemeinschaft zu stiften:

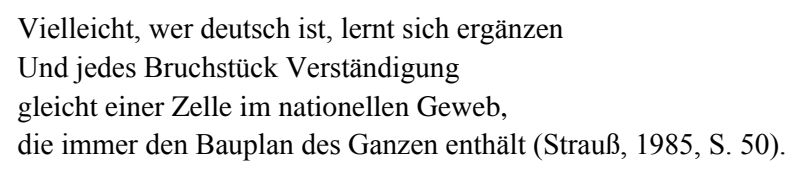

Diese mythisierende, weil hinter die Alltagspolitik auf eine höhere Sphäre der Dichtung zurückverweisende Klage über die auch individuell zu erfahrende Teilung der einen Nation in zwei einander feindliche Staaten hat seinerzeit noch zu provozieren vermocht, weil sich das Pathos ihres evident artifiziellen Sprechens gar zu nachdrücklich dem allseitigen Konsens faktischer Koexistenz entzog und einen überhistorischen Ernst anmahnte, bei dem es sich nicht leicht bequem machen ließ. Weniger provokant hat gut zwei Jahre zuvor Peter Schneiders Der Mauerspringer (1982) gewirkt. Diese im Untertitel als 'Erzählung' ausgewiesene Vermischung von faktenbezogenem Essay, autobiografischer Selbstreflexion und fiktionaler Ausgestaltung setzt mit der sinnlichen Erfahrung ein, dass ein von West her kommendes Flugzeug, das in Berlin-Tegel landen will, das „,raumaufteilende Bauwerk“ der Berliner Mauer „dreimal überqueren“ muss und den Passagieren dennoch „einen durchaus einheitlichen Anblick“ der in Wahrheit so gründlich zerrissenen Stadt bietet: „Nichts bringt den Ortsunkundigen auf die Idee, daß er sich einer Gegend nähert, in der zwei politische Kontinente aufeinanderstoßen" (Schneider, 1982, S. 5). Diese Unwahrnehmbarkeit der Mauer gibt das Leitmotiv des Erzählessays vor, der seinen Ausgangspunkt bei der Erkenntnis nimmt, das „massive Ding“ habe sich „im Bewußtsein der Westdeutschen immer mehr zur Metapher“ entwirklicht: „Was jenseits das Ende der Bewegungsfreiheit bedeutete, wurde diesseits zum Sinnbild für ein verabscheutes Gesellschaftssystem (...): die Mauer wurde den Deutschen im Westen zum Spiegel, der ihnen Tag für Tag sagt, wer der Schönste im Lande ist“" (Schneider, 1982, S. 13).

In weitgehender Übereinstimmung mit dem Lebenslauf Peter Schneiders (1940 in Lübeck geboren), der sich früh den Ruf eines schriftstellerischen Repräsentanten 
der 68er-Generation erworben hat, reflektiert der Ich-Erzähler seine mittlerweile 20-jährige Erfahrung mit der „siamesischen Stadt“, zu deren kasernenartiger Rechtwinkligkeit „die Mauer in ihrem phantastischen Zickzackkurs“ überhaupt nicht passen will, im Kontrast vielmehr „wie die Ausgeburt einer anarchischen Phantasie wirkt" und daher „eher als städtebauliches Kunstwerk denn als Grenze“ erscheint (Schneider, 1982, S. 6 f.). Die analoge Beobachtung an Gesprächen im Schwarzwald wie in Dresden oder Leipzig, ,je weiter weg von der Grenze, desto ungenierter“ bilde „das jeweils halbe Volk sich ein, ein ganzes zu sein“, hat dem Wahl-Berliner bewusst werden lassen, dass er die Teilung der Stadt im Alltagsleben gar nicht mehr bemerkt: „Tatsächlich sehe ich die Mauer nicht mehr, obwohl sie nächst der chinesischen das einzige Bauwerk auf der Erde sein dürfte, das sich vom Mond aus mit bloßem Auge erkennen läßt" (Schneider, 1982, S. 9). Umso mehr leitet er daraus sein Projekt ab, „Geschichten über die geteilte Stadt zu sammeln“ (Schneider, 1982, S. 21), das bei dem befreundeten Ex-Ostberliner Poeten Robert allerdings auf Skepsis stößt, weil dieser nach seiner Übersiedelung in den Westteil der Stadt schnell gelernt hat, sich dem falschen Interesse an seiner Vita zu entziehen. Der Erzähler mag sich allerdings nicht mit dem Faktum abfinden, dass die 'deutsche Frage' „,in dreißig Jahren Speck angesetzt" hat und die Teilung für „die meisten Deutschen westlich der Elbe“ langst Normalität geworden ist: „Nicht die Empfindung einer unerträglichen Situation hat mich dazu gebracht, sondern das Mißtrauen in die Abwesenheit einer solchen Empfindung“" (Schneider, 1982, S. 21).

Dieser Gedankenlosigkeit werden mehrere Geschichten von 'Mauerspringern' entgegengehalten, die den Hauptteil der 'Erzählung' ausmachen und wenigstens zum Teil auf historischen Fakten beruhen, wie immer sie sich „unentwirrbar“ untereinander vermischen mögen (Schneider, 1982, S. 46). In stillschweigendem Anschluss an Stefan Heyms Erzählung Mein Richard (1974), die zwei Ostberliner Jugendliche insgesamt 14-mal zum Kino-Gang in den Westen wechseln lässt, erfindet Peter Schneider die Geschichte von Lutz und zwei Freunden des gleichen Namens Willy, die insgesamt zwölf Mal springen (Schneider, 1982, S. 53), durch einen westdeutschen Journalisten unbeabsichtigt denunziert werden und trotz des Arguments ihres Verteidigers, die zwölfmalige Rückkehr sei „ein Treuebeweis, dessen sich nur wenige DDR-Bürger rühmen könnten“ (Schneider, 1982, S. 53), die Härte der DDR-Gerichtsbarkeit zu spüren bekommen. Obwohl sich der Erzähler zunächst selbst ins Wort fällt, „denn genau so, wie ich den Grenzübertritt der drei Kinogänger wiedergegeben habe, kann er sich kaum abgespielt haben“ (Schneider, 1982, S. 50), fährt er mit seiner Darstellung fort, da die Tatsachen ,im Gestrüpp der mündlichen Überlieferung“ ohnehin „untergegangen“ sind und die „Phantasie derer“, „die die ganze Geschichte weiterverbreiteten" (Schneider, S. 52), längst alle faktischen Schwierigkeiten gelöst hat. Umso zwangloser kann Peter Schneiders Alter Ego diese fiktionale Episode mit den historisch belegten Sprüngen des Westberliner Sozialhil- 
feempfängers Arnold Kabe vermengen, dessen 15-maliges Überschreiten der Grenze einst ,zu einer ernsten Belastung für die deutsch-deutschen Beziehungen“ (Schneider, 1982, S. 34) geworden ist (vgl. die Darstellung des Falles in: Schaad, 2009, S. 128-136). In einem Gedankenspiel kommt es an einem Nebeltag schließlich zur Ost-West-Kollision auf der Mauer:

Kabe hangelt sich am Seil empor und will eben zu seinem 15. Sprung ansetzen, während Lutz und die beiden Willy Schwung zum 12. holen. Der Nebel behindert die Sicht derart, daß Kabe dem blind springenden Lutz erst im letzten Moment ausweichen kann. Unfreiwillig umarmen sich die Kollegen, damit sie nicht gemeinsam auf einer Seite der Mauer herabfallen, die entweder für Kabe oder für Lutz die falsche wäre. (Schneider, 1982, S. 56 f.)

Ein Gespräch über die Motive zum Springen müsste allerdings schnell ,,ins Stocken geraten“, weil es „kaum interessanter“ wäre „,als die Unterhaltung zweier Seilschaften, die sich unerwartet auf dem K 2 begegnen und die schöne Aussicht als Grund für den lebensgefährlichen Aufstieg angeben“ (ebd.).

Indem mit der wiederum authentischen "Geschichte von Walter Bolle, der auf eigene Faust Krieg gegen die DDR führen wollte" (Schneider, 1982, S. 80), und der tragisch geendeten Vita Michael Gartenschlägers (getötet 1976 beim Versuch, einen Selbstschuss-Automaten der DDR abzubauen) noch weitere Versionen deutschdeutschen Aberwitzes zur Darstellung kommen, tritt die Erzählabsicht umso deutlicher hervor: Dass ,östlich der Elbe“ das 'Gespenst' des Kommunismus, wie es Karl Marx und Friedrich Engels im Manifest der kommunistischen Partei (1848) einleitend beschworen haben, buchstäblich „seßhaft geworden“ ist und „tatsächlich zum Fürchten“ aussieht, ist eine historische Wahrheit, an deren Anblick sich selbst ,politische Exorzisten“ so sehr gewöhnt haben, dass sie sich „nur noch an Feiertagen erschreckt" geben (Schneider, 1982, S. 30). Die belegten resp. unbelegten Anekdoten, die Peter Schneiders Der Mauerspringer versammelt und an die subjektivautobiografischen Erfahrungen des Ich-Erzählers bindet, haben demgegenüber das Potenzial, der Gewöhnung an das Widersinnige zu wehren. Sie arbeiten vor allem deswegen der notwendigen Sensibilisierung zu, weil der Selbstbeobachtung des IchErzählers längst deutlich geworden ist, dass die „Mauer im Kopf“ dauerhafter ist als die „sichtbare“ Mauer (Schneider, 1982, S. 117). Es sind nicht allein die Medien des Ostens wie des Westens, die je auf ihre Weise einseitig berichten und hinsichtlich der polnischen Streik-Bewegung wahlweise antisozialistische Umtriebe bzw. Unterdrückung von Regierungsseite brandmarken (Schneider, 1982, S. 115). Auch auf persönlich-privater Ebene ist die deutsch-deutsche Spaltung prägend und bringt eine „Art der Zugehörigkeit“ mit sich, „die sich jenseits jeder politischen Option durchsetzt“, sodass der Ich-Erzähler und seine westöstlichen Gesprächspartner „nicht miteinander reden“ können, „ohne daß ein Staat aus uns spricht" (Schneider, 1982, S. 117). Diese Reflexion mündet in die abschließende Grundsatzüberlegung, inwiefern sich Deutschland angesichts der beiden Staaten überhaupt noch als 'Vaterland' 
verstehen lässt: „Falls mein Vaterland existiert, so ist es kein Staat, und der Staat, dessen Bürger ich bin, ist kein Vaterland“.

'Deutschland' kann es deshalb auf keiner politischen Landkarte mehr geben, sondern nur in seiner Geschichte und seiner Sprache: „Und wie vor 1000 Jahren kann der Versuch, eine gemeinsame deutsche Sprache zu sprechen, nur mit einer Weigerung anfangen: mit der Weigerung, das Kirchenlatein aus Ost und West nachzuplappern“. Peter Schneiders Erzählung Der Mauerspringer stellt einen solchen Versuch dar, 'deutsch' zu sprechen und in diesem Sprechen im Widerstreit mit der politischen Realität doch die „Zugehörigkeit zu einem Volk“ zur Geltung zu bringen, die als 'nationale Identität' nicht an die „Zugehörigkeit zu einem der beiden deutschen Staaten gebunden ist" (Schneider, 1982, S. 124 f.). Die Erzählung selbst aber endet für ihren Erzähler eher pessimistisch-melancholisch mit der nicht begründeten Verweigerung seiner Einreise in die DDR, wodurch das eigene, im Rahmen der Legalität vielfach praktizierte 'Mauerspringen' drastisch unterbunden wird, und mit der subjektiven Besinnung auf das Vergehen der Zeit: „Nur die Stadt drauBen mit ihren Brandmauern, Hinterhofmauern, Grenzmauern - diese Mauern werden noch stehen, wenn niemand mehr da sein wird, der hindurchgehen könnte" (Schneider, 1982, S. 135).

Im Unterschied zu diesem poetisch ausgeschmückten Essay, der seine entscheidenden Motive gleichermaßen erzählerisch wie begrifflich auf den Punkt bringt, führt Dorle und Wolf des einstigen DKP-Sympathisanten Martin Walser (geb. 1927) im rein fiktionalen Medium die Unerträglichkeit deutsch-deutscher Zweistaatlichkeit vor Augen. 1987, nicht lange vor Mauerfall und Beitritt der DDR zur Bundesrepublik, greift die im Untertitel als 'Novelle' ausgewiesene Erzählung die um die Mitte der 1980er Jahre zum Skandal gewordene, jedoch schon seit Jahrzehnten gängige Praxis der Staatssicherheit auf, männliche Agenten ('Romeos') auf Mitarbeiterinnen westdeutscher Ministerien anzusetzen, um sie erotisch abhängig zu machen und zum Geheimnisverrat zu motivieren (vgl. Pfister, 1999). Wolf Zieger - einleitend zum Major der Nationalen Volksarmee befördert - ist ehrlich von der Notwendigkeit überzeugt, die vielfältige Überlegenheit der BRD durch Spionage auszugleichen, und wird zum einen in seiner Agentenkarriere, zum anderen in seinen daraus folgenden Privatverwicklungen geschildert: Vor 15 Jahren aus der DDR übersiedelt, hat er vor neun Jahren Dorle, eine Sekretärin des Bundesverteidigungsministeriums, geheiratet und ihr nach der Hochzeit seine Verpflichtung durch die Staatssicherheit gestanden; parallel zur Ehe pflegt er eine der Gattin bekannte Affäre mit ihrer Kollegin Sylvia, um lohnendes Material abschöpfen zu können. Zunehmend wird Wolf jedoch der Problematik dieses Handelns inne. Zwar glaubt er an seinen Beitrag zur Vermeidung des 'Ernstfalls', kann das aber nicht mehr so ernst nehmen wie einst: „Wir verhindern ihn, Dorle, sagte Wolf. | Du, sagte Dorle. | Du und ich, sagte Wolf, per Parodie. Mehr ist es ja nicht, was wir zwischen diesen beiden verrannten Deutsch-Hälften tun“(Walser, 1987, S. 68). 
Zentral ist in diesem Zusammenhang die von allen Rezensenten der Neuerscheinung herausgehobene Passage am Hauptbahnhof von Bonn, als die anderen Reisenden Wolf plötzlich „wie halbe Menschen“vorkommen:

\footnotetext{
Lauter Halbierte strebten da hin und her. Die anderen Hälften liefen in Leipzig hin und her. Die hier leuchteten, gleißten geradezu in ihrer Entwickeltheit und Fortgerissenheit. Er fühlte sich hingezogen zu allen. Wie richtig machten die alles, was sie machten! Aber wie wenig waren sie bei sich. Alle leuchteten vor Gelungenheit, aber keiner schien zufrieden zu sein. Sie wissen nicht, was ihnen fehlt. Und keiner würde, fragte man ihn, sagen, ihm fehle seine Leipziger Hälfte, sein Dresdener Teil, seine mecklenburgische Erstreckung, seine thüringische Tiefe. Aber sie sind wie verloren in ein Extrem. Und die drüben sind verrannt ins andere Extrem. Das teilt mehr als der böse Strich durch die Geographie. (Walser, 1987, S. 54 f.)
}

Dass Wolf als ausgebildeter Pianist die Novelletten Robert Schumanns immer nur mit einer Hand spielt (Walser, 1987, S. 30), weist als novellentypisches Symbol in die gleiche Richtung der deutsch-deutschen Teilung als unnatürliche Halbierung bzw. als (Selbst-)Amputation.

Martin Walsers 'Romeo', dessen private Doppelung zwischen Dorle und Sylvia seiner politischen zwischen den konkurrierenden Staaten entspricht, stellt sich schließlich den Behörden der Bundesrepublik. Im Gefängnis fasst er den Entschluss, jeden Tag an die - zwischenzeitlich endlich schwangere - Ehefrau Dorle zu schreiben: „Bis zur Erschöpfung. Er würde ihr sein Leben erzählen. Zum ersten Mal“ (Walser, 1987, S. 172). Die Erzählung selbst lässt sich insofern als eine in die Dritte Person objektivierte Beichte verstehen, wodurch das Erzählen retrospektiv doch noch eine Art von 'novellistischer' Rahmung gewinnt. Im Schreiben wird Wolf seine Gespaltenheit überwinden können und eben zu derjenigen Identität mit sich selbst finden, die ihm - wie unbewusst allen anderen auch - bislang gefehlt hat: „Liebes Dorle, schrieb er, und schon bei dieser einfachen Anrede hatte er das Gefühl, als starte er, als hebe er ab und werde lange nicht mehr landen“" (Walser, 1987, S. $176 \mathrm{f}$.).

Sinnfälliger als im surrealen Bild der halben Menschen hier wie dort ist die Trennung der Deutschen von den Deutschen nirgendwo zum Ausdruck gekommen: „Aber er wunderte sich, warum es keiner ausrief: Wir sind Halbierte. Und er am meisten" (Walser, 1987, S. 55). Am Beispiel des privat wie dienstlich in die Abgründe der politischen Gegebenheiten verstrickten Wolf Zieger, dem es anders als den Anderen immer weniger gelingt, das Anormale als Normales hinzunehmen, geht es Martin Walser um die Sensibilisierung seiner Leser für die Absurdität der politischen Teilung, mit der man sich gerade aus der freilich bequemen Perspektive der prosperierenden Bundesrepublik bedenklich leichtfertig als nicht weiter störendes Faktum abgefunden hat.

Dass es so bald nach Dorle und Wolf zur politischen Wiedervereinigung kommen sollte, bestätigt kaum Ernst Blochs Glauben an die Kraft der Literatur, als 'Vorschein' (Jung, 2012) realer Entwicklung der Empirie vorauszueilen. Die Thematisie- 
rung der deutschen Teilung durch ehemals 'linke' Autoren der Bundesrepublik dürfte vielmehr darauf verweisen, dass die literarische Verdrängung der historischen Tatsachen im Laufe der 1980er Jahre angesichts der Gewaltsamkeit so sehr zum Unding geworden ist, dass auch mit den Mitteln der Dichtung darauf zu reagieren war, wie marginal dies auch immer geschah. Ab 1989 hat die Realgeschichte dann ohnehin keinen Anlass mehr geboten, die deutsche Teilung poetisch zu reflektieren.

\section{Bibliographie}

Barthes, R. (2002). L'effet de réel. In Ders. Euvres complètes. Tome III : 1968-1971. Nouvelle édition revue, corrigée et présentée par Éric Marty (S. 25-32). Paris: Seuil.

Fuld, W. (13. Juli 1985). Wanderprediger der neuen Mystik. Botho Strauß' Gedicht ,Die Erinnerung an einen, der nur einen Tag zu Gast war'. Frankfurter Allgemeine Zeitung, S. 24.

Grundmann, V. K. (1988). Das geteilte Deutschland. Eine literarische Thematik im Wandel der politischen Verhältnisse, dargestellt an ausgewählten Beispielen der Prosa der Deutschen Demokratischen Republik. 1949-1984. Buffalo: University Microfilms International.

Hoffmann, T. (2006). Konfigurationen des Erhabenen. Zur Produktivität einer ästhetischen Kategorie in der Literatur des ausgehenden 20. Jahrhunderts (Handke, Ransmayr, Schrott, Strauß). Berlin: De Gruyter.

Hutchinson, P. (2011). Literary Presentations of Divided Germany. The Development of a Central Theme in East German Fiction. 1945-1970. Cambridge: University Press [zuerst 1977 erschienen].

Jung, W. (2012). Vor-Schein. In B. Dietschy, D. Zeilinger, R. \& E. Zimmermann (Hrsg.), BlochWörterbuch. Leitbegriffe der Philosophie Ernst Blochs (S. 664-672). Berlin: De Gruyter.

Kilian, W. (2001). Die Hallstein-Doktrin. Der diplomatische Krieg zwischen der BRD und der DDR 1955-1973, aus den Akten der beiden deutschen Außenministerien Berlin: Duncker \& Humblot (Zeitgeschichtliche Forschungen, 7).

Lindenberg, U. (2002): Wir wollen doch einfach nur zusammen sein (Mädchen aus Ost Berlin). Beiheft zu: U. Lindenberg \& Das Panik Orchester: Alles klar auf der Andrea Doria (o. S.). eastwest records.

Meier, A. (1998). „dieser Messerschnitt entlang der Elbe”. Bundesrepublik und DDR in zeitkritischen Nachkriegsromanen. In H. Detering \& H. Krämer (Hrsg.), Kulturelle Identitäten in der deutschen Literatur des 20. Jahrhunderts (S. 59-68). Frankfurt a. M.: Peter Lang.

Meier, A. (2003). Pessimismus von links. Wolfgang Koeppens Tauben im Gras im Kontext des bundesrepublikanischen und italienischen Nachkriegsromans. Jahrbuch der Internationalen Wolfgang Koeppen-Gesellschaft, 2, 135-149.

Meier, A. (2006). „Ich lebe vom Schreiben“. Wolfgang Koeppens Poetik. Treibhaus. Jahrbuch für die Literatur der fünfziger Jahre, 2, 202-216.

Pfister, E. (1999). Unternehmen Romeo. Die Liebeskommandos der Stasi. Berlin: Aufbau.

Schaad, M. (2009). „Dann geh doch rüber“. Über die Mauer in den Osten. Berlin: Ch. Links.

Schmidt, A. (1956). Das steinerne Herz. Historischer Roman aus dem Jahre 1954. Karlsruhe: Stahlberg.

Schneider, P. (1982). Der Mauerspringer. Erzählung. Darmstadt: Luchterhand.

Strauß, B. (1985). Diese Erinnerung an einen, der nur einen Tag zu Gast war. Gedicht. Mit einer Nachbemerkung von Martin Walser. München: Carl Hanser.

Strauß, B. (1990). Der Aufstand gegen die sekundäre Welt. Bemerkungen zu einer Ästhetik der Anwesenheit. In: G. Steiner. Von realer Gegenwart. Hat unser Sprechen Inhalt? Mit einem Nachwort von Botho Strauß (S. 315-316) (J. Trobitius Übers.). München: Carl Hanser.

Walser, M. (1987). Dorle und Wolf. Eine Novelle. Frankfurt a. M.: Suhrkamp. 